\title{
EFEITO DO TIPO DE FERMENTAÇÃO NA QUALIDADE FINAL DE EMBUTIDOS FERMENTADOS COZIDOS ELABORADOS A BASE DE CARNE OVINA
}

\author{
ROSALI AMARAL MATOS* \\ CARINE MOTA MENEZES* \\ EDUARDO MENDES RAMOS** \\ ALCINÉIA DE LEMOS SOUZA RAMOS*** \\ LÚCIO ALBERTO DE MIRANDA GOMIDE****
}

\begin{abstract}
No presente trabalho avaliou-se o efeito da fermentação controlada pelo uso de cultura iniciadora e da fermentação induzida pela adição de gluconadelta-lactona (GDL) na qualidade de embutidos fermentados cozidos, elaborados com carne ovina. $\mathrm{O}$ embutido adicionado de GDL que apresentava $\mathrm{pH}$ próximo de 5,6 alcançou pH 5,3 logo após a mistura dos ingredientes à massa carnica. Já com o uso de culturas iniciadoras foi necessário tempo de fermentação maior que 10 horas para alcançar esse pH. Após 26 horas de fermentação, a massa adicionada de GDL apresentou $\mathrm{pH}$ 4,81 e acidez de $1,03 \%$ e a massa adicionada de cultura revelou $\mathrm{pH}$ final 4,37 e acidez de 1,26\%. Os produtos acabados evidenciaram atividade de água entre 0,92 (GDL) e 0,93 (cultura). Diferentemente do índice de amarelo, maior $\left(b^{\star}=8,62\right)$ nos embutidos adicionados de GDL que nos adicionados de cultura $\left(b^{\star}=7,75\right)$, os índices de luminosidade $\left(L^{*}=46,80\right)$ e de vermelho $\left(a^{*}=12,54\right)$ não foram afetados pelo tipo de fermentação utilizada. Os embutidos adicionados de cultura apresentaram maior dureza (20,38 contra $17,31 \mathrm{kgf})$ e resistência ao corte (1,50 contra $1,16 \mathrm{kgf} / \mathrm{cm})$, o que indica melhor consistência (firmeza) do que os embutidos adicionados de GDL.
\end{abstract}

PALAVRAS-CHAVE: FERMENTAÇÃO; SALAME; EMBUTIDOS; CARNE OVINA.

* Engenheiras de Alimentos, formadas pela Universidade Estadual do Sudoeste da Bahia (UESB), Itapetinga, BA.

** Doutor em Ciência e Tecnologia de Alimentos, Professor Adjunto, Departamento de Ciência de Alimentos, Universidade Federal de Lavras (DCA/UFLA), Lavras, MG (e-mail: emramos@ufla.br).

*** Mestre em Ciência e Tecnologia de Alimentos, Professora Assistente, Departamento de Tecnologia Rural e Animal, Universidade Estadual do Sudoeste da Bahia (DTRA/UESB), Itapetinga, BA (e-mail: alcineia@uesb.br).

**** Ph.D. em Food Science, Professor Adjunto, DTA, UFV, Viçosa, MG (e-mail: lagomide@ufv.br). 


\section{INTRODUÇÃO}

O consumo per capta anual da carne ovina/caprina no Brasil foi estimado em $0,70 \mathrm{~kg}$, pouco representativo quando comparado com países desenvolvidos (20 a $28 \mathrm{Kg}$ ), ou ao consumo brasileiro de carnes bovina (36 kg), suína (10 kg) e de frango (24 kg) (SEBRAE/CE, 1998). Em coleta de dados, realizada em 2003, foi estimado em Fortaleza (CE) consumo per capita anual de $0,590 \mathrm{~kg}$ para a carne ovina e de $0,375 \mathrm{~kg}$ para a carne caprina, totalizando 0,965 $\mathrm{kg}$ per capita/ano (SEBRAE/CE, 2003). O consumo total de carne ovina/caprina na cidade de Natal (RN) foi estimado em 0,897 kg per capita/ano, sendo 0,430 kg para a carne ovina e de 0,467 kg para a carne caprina (SEBRAE/RN, 2001). Já MOREIRA et al. (1997) constataram consumo per capita anual de 10,81 kg em Juazeiro (BA) e $11,73 \mathrm{~kg}$ em Petrolina (PE), representando em torno de 2.300 toneladas/ano. Esse elevado consumo anual de carne ovina/caprina nas cidades de Fortaleza, Natal, Juazeiro e Petrolina contrasta com as 300 toneladas anuais estimadas para o Distrito Federal, que representa consumo de apenas $0,150 \mathrm{~kg}$ per capita/ano (MEDEIROS et al., 2004). Tal fato demonstra o elevado grau de condicionamento cultural na alimentação do nordestino no que se refere ao consumo de carnes caprina e ovina.

Se o consumo de carne ovina/caprina se mostra significativamente superior no eixo nortenordeste, isso se deve mais à demanda reprimida (insuficiência na oferta) nos demais centros que propriamente da necessidade de introdução de novos hábitos de consumo (MEDEIROS et al., 2004). Existe, portanto, grande espaço para a expansão do consumo de carne ovina/caprina no mercado brasileiro de carnes.

Tem sido observada maior procura por carne in natura de caprinos e ovinos, sendo que cortes nobres alcançam bons valores no mercado. O preconceito envolvendo o consumo dessas carnes nos centros urbanos é pequeno em relação ao universo de consumidores, não havendo expansão da procura por esses produtos devido à sua oferta limitada e irregular (CAVALCANTI e SILVA, 1988). O processamento dessas carnes permite agregar valor aos cortes não-aproveitados para o consumo in natura, gerando empregos e aumentando a receita e a oferta de produtos disponíveis no mercado.

BERRY, CROSS e SMITH (1990) compararam produtos reestruturados elaborados com carne de caprinos e de outros tipos animais. Afirmaram que a rejeição aos produtos contendo carne de caprinos, aparentemente devido ao aroma e sabor característicos, poderia ser revertida com uso de carne bovina ou suína em formulações mistas. A utilização de carnes caprinas ou ovinas em formulações de embutidos, juntamente com carnes bovinas e suínas, foi uma das alternativas tecnológicas propostas por ZAPATA (1994). A elaboração de embutidos fermentados representa alternativa viável de processamento por se tratar de produto estável em temperatura ambiente, o que facilita sua comercialização e permite alcançar novos mercados consumidores.

Embutidos fermentados são, convencionalmente, preparados a partir da mistura de carnes bovina e suína moídas em diferentes proporções, com variações quanto à composição e adição de condimentos. Diferencia-se dos demais embutidos pela elevada presença de ácido lático, que the confere sabor característico e pelos baixos teores de umidade e baixos valores de atividade de água. Podem ser classificados como secos ou semi-secos, dependendo da quantidade de água perdida durante o processo (AMIF, 1997). Os embutidos semi-secos normalmente são defumados e submetidos a temperatura de pelo menos $63^{\circ} \mathrm{C}$ antes da secagem, enquanto que os secos não são cozidos e podem ser defumados ou não (PEARSON e GILLETT, 1999).

Para favorecer as condições de produção de alimentos fermentados seguros, o Food Safety Inspection Service (FSIS-USDA) recomenda: a fermentação deve ocorrer de forma que o pH 5,3 (ou menos) seja alcançado em certo intervalo de tempo para inibir o crescimento de determinadas bactérias patógenas (como a Escherichia coli e o Staphylococcus aureus). No 
caso da presença da E. coli O157:H7, a redução do $\mathrm{pH}$ para valores abaixo de 5,3 tem sido considerada insuficiente para inativa-la, sendo aconselhada etapa de cozimento para sua destruição e garantir a segurança alimentar do produto (AMIF, 1997).

Salames semi-secos podem ser preparados de forma segura com tempo de fermentação inferior a 24 horas, exigindo controle cuidadoso da temperatura e utilização de culturas iniciadoras ou agentes acidulantes (BUSBOOM e FIELD, 2003). As culturas são adicionadas rotineiramente na massa do salame para se obter contagem microbiana mínima de $10^{6}$ organismos por grama do produto. Esse número inicial geralmente assegura a dominância das culturas láticas sobre microrganismos indesejáveis. O uso de acidulantes químicos, como a glucona-delta-lactona (GDL) e ácidos (lático ou cítrico) encapsulados, inibem o desenvolvimento de microrganismos indesejáveis pela rápida queda do $\mathrm{pH}$ da massa cárnica, estimulando o desenvolvimento das bactérias láticas (FREY, 1983).

No presente trabalho analisou-se a etapa de fermentação de embutidos cozidos a base de carne ovina, comparando os processos de fermentação induzida quimicamente (adição de GDL) e de fermentação controlada pela adição de culturas iniciadoras (culturas starters), além da avaliação dos produtos acabados.

\section{MATERIAL E MÉTODOS}

\subsection{PREPARO DOS EMBUTIDOS}

Foram utilizadas carnes da paleta de ovinos, acém bovino e toucinho suíno, provenientes de animais adultos sem distinção de raça ou castração, adquiridas no mercado de Itapetinga (Bahia). As carnes foram individualmente moídas em discos de $8 \mathrm{~mm}$, misturadas e adicionadas dos ingredientes indicados na Tabela 1. Na fermentação induzida quimicamente foram adicionados 0,5\%, em relação à massa cárnica, de glucona-delta-lactona (Dairy Mix GDL, Germinal) e 1,5\% de vinho tinto seco. Na fermentação controlada foram adicionados 1,5\% de glicose e 1,45\% de cultura iniciadora liofilizada (Germinal) para maturação de salames, composta de Staphylococcus carnosus e Pediococcus pentosaceus.

\section{TABELA 1 - FORMULAÇÃO BASE DOS EMBUTIDOS COM CARNE OVINA}

\begin{tabular}{lc}
\hline Massa Cárnica & Quantidade \\
\hline Carne (paleta) ovina & $60 \%$ \\
Carne (acém) bovina & $20 \%$ \\
Toucinho suíno & $20 \%$ \\
\hline Ingredientes & Quantidade* \\
\hline New Mix Salame - 759 (New Max Industrial) & $2,0 \%$ \\
Leite em pó & $3,0 \%$ \\
Pó Húngaro & $0,3 \%$ \\
\hline
\end{tabular}

* em relação à massa cárnica.

As misturas de carnes e ingredientes foram embutidas em tripa celulósica de $45 \mathrm{~mm}$ de diâmetro, formando gomos de aproximadamente $15 \mathrm{~cm}$. Os embutidos foram, então, pesados e conduzidos para fermentação em câmara BOD, regulada para temperatura de $35^{\circ} \mathrm{C}$. Após 26 horas de fermentação, os embutidos foram submetidos ao cozimento em estufa a $60 \pm 5^{\circ} \mathrm{C} / 1$ hora, seguido de 
$70 \pm 5^{\circ} \mathrm{C} / 1$ hora e aquecimento a $80 \pm 5^{\circ} \mathrm{C}$ até atingir $71^{\circ} \mathrm{C}$ no centro da peça. Alcançada a temperatura de cozimento, as peças foram imediatamente resfriadas com borrifos de água gelada para cessar o aquecimento do produto. Os embutidos cozidos foram armazenados em temperatura de refrigeração $\left(4^{\circ} \mathrm{C}\right)$ por 10 dias para secagem parcial, quando o produto foi considerado acabado.

\subsection{ANÁLISES FÍSICAS E QUÍMICAS}

Durante a etapa de fermentação foram avaliados o pH e a acidez dos embutidos nos tempos zero, 3, 6, 20, 23 e 26 horas. Uma amostra de 10 gramas do embutido foi triturada e homogeneizada em $100 \mathrm{~mL}$ de água destilada por 60 segundos, sendo a leitura do pH realizada com eletrodo de vidro após imersão por 5 minutos no homogenato. Logo após a leitura do pH determinou-se a acidez do homogenato expressa em ácido lático mediante titulação colorimétrica, utilizando-se a fenolftaleina como indicador (MATOS et al., 2005b)

A atividade de água $\left(\mathrm{a}_{\mathrm{w}}\right)$ dos produtos acabados foi medida pela técnica do ponto de orvalho em espelho resfriado (AQUALAB, 1997), utilizando-se o aparelho AQUALAB-Decagon, modelo Cx2T, com resolução de $0,01 a_{w}$ e operado na temperatura de $26 \pm 1^{\circ} \mathrm{C}$.

A avaliação objetiva da cor final dos embutidos foi realizada em colorímetro ColorQuest II Sphere (Hunterlab, Reston, VA), conectado em computador equipado com o programa Universal ${ }^{\circledR}$. Para medida dos índices de cor foi estabelecido o iluminante $A$ e o ângulo de $10^{\circ}$ para o observador, sendo utilizado o sistema de cor CIELAB. As análises foram conduzidas com a reflectância especular incluída (RSIN). Os índices de cor $L^{*}, a^{*}$ e b* foram obtidos considerando-se o valor médio de 6 leituras realizadas na superfície de fatias do produto de aproximadamente $2 \mathrm{~cm}$ de espessura. A saturação $\left(C^{\star}\right)$ e o ângulo de tonalidade $\left(h^{\star}\right)$ foram calculados pelas seguintes fórmulas (AMSA, 1995): $C^{\star}=\left[\left(a^{\star}\right)^{2}+\left(b^{\star}\right)^{2}\right]^{1 / 2}$ e $h^{\star}=\tan ^{-1}\left(b^{\star} / a^{\star}\right)$.

As análises de textura nos produtos acabados foram conduzidas em texturômetro TA.HDI Texture Analyser (Stable Micro Systen Inc.), conectado em computador equipado com o programa Texture Expert ${ }$. Foram aplicados dois métodos: o teste de força de cisalhamento e a análise de perfil de textura (APT). No teste de cisalhamento, as amostras foram cortadas em cilindros de $4 \mathrm{~cm}$ de altura e a tripa celulósica removida. Conduziu-se o cisalhamento de forma transversal, a $2 \mathrm{~cm}$ das extremidades, por lâmina plana sem fenda (HDP/B5 Stable Micro Systen Probe). Usou-se velocidade de teste de $3 \mathrm{~mm} / \mathrm{s}$ e a de pré e pós-teste de $10 \mathrm{~mm} / \mathrm{s}$, sendo a razão entre a força máxima e o diâmetro do embutido $(\mathrm{kgf} / \mathrm{mm})$ relacionada à resistência ao corte. No teste de APT, as amostras foram cortadas em cilindros com $2 \mathrm{~cm}$ de altura e comprimidos duas vezes até $60 \%$ de seu tamanho por cilindro de $50 \mathrm{~mm}$ de diâmetro (SMSP/50 Stable Micro Systen Probe). Não houve tempo de repouso entre os dois ciclos de compressão. A curva de deformação com o tempo foi obtida com velocidade de compressão de $5 \mathrm{~mm} / \mathrm{s}$, a partir da qual foram gerados os seguintes parâmetros de textura: fraturabilidade, dureza, coesividade, adesividade, elasticidade e mastigabilidade (BOURNE, 1978; SZCZESNIAK, 1998).

\section{RESULTADOS E DISCUSSÃO}

A Figura 1 ilustra a taxa de queda do pH e o concomitante aumento na produção de ácidos nos embutidos avaliados.

O decréscimo do $\mathrm{pH}$ ocorreu a partir de 6 horas na massa adicionada de cultura iniciadora, assim como observado por ANDRADE et al. (2005) na elaboração de embutido cozido a base de carne suína. Esse período de retardo, também observado na curva de acidez, pode ser oriundo da necessidade das culturas liofilizadas absorverem umidade e voltarem à forma vegetativa antes de começarem a produzir ácido, bem como de sua adaptação às condições na massa cárnica. 


\section{FIGURA 1 - CURVA DE pH E PERCENTUAL DE ÁCIDO LÁTICO DURANTE A FERMENTAÇÃO $\left(35^{\circ} \mathrm{C}\right)$ DE EMBUTIDOS ADICIONADOS DE CULTURA INICIADORA E GDL}

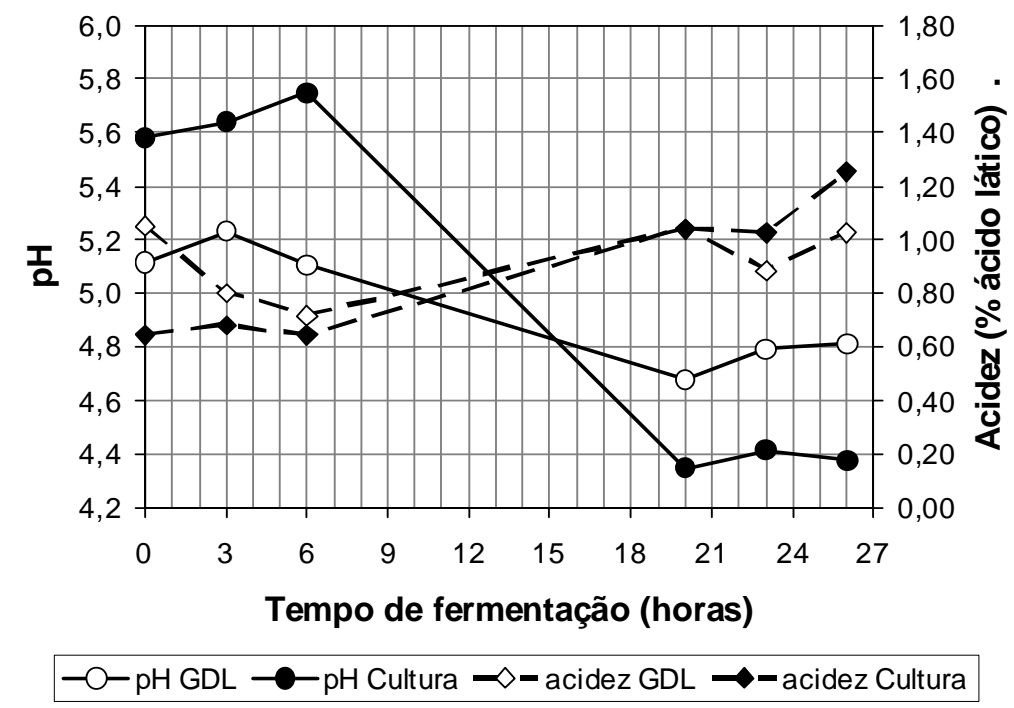

A queda do $\mathrm{pH}$, induzida pela cultura iniciadora, foi muito mais rápida que o intervalo de 32 a 40 horas citado por EVERSON, DANNER e HAMMES (1970) como tempo médio de fermentação para culturas liofilizadas. Ao acompanhar a fermentação de embutido semi-seco a base de carne de peru, KELLER e ACTON (1974) constataram tempo de aproximadamente 18 horas para que fosse alcançado pH 5,3 em embutido adicionado de cultura iniciadora (Pediococcus cerevisiae) liofilizada. Já ANDRADE et al. (2005), utilizando cultura liofilizada (Lactobacillus plantarum) em embutido suíno, observaram tempo médio de 14 horas. Tal tempo foi considerado curto por esses autores quando comparado aos 3 a 4 dias necessários para a obtenção de salames de fermentação rápida. MATOS et al. (2005a) compararam a fermentação natural (sem inóculo) com o uso de iogurte natural na elaboração de embutidos cozidos a base de carne ovina. Observaram que o início da queda de $\mathrm{pH}$ ocorreu apenas depois de 24 horas de fermentação, sendo que pH inferior a 5,4 não foi verificando mesmo após 48 horas de fermentação.

No caso do uso da glucona-delta-lactona (GDL) como acidulante, a rápida queda de $\mathrm{pH}$ pode ser oriunda não apenas da conversão da GDL a ácido glucônico, mas também da adição de vinho à massa que contribui com a presença de ácidos orgânicos pré-formados. Segundo FREY (1983) e SAVIC (1985), dependendo da quantidade de GDL adicionada e da temperatura de fermentação utilizada, a acidificação da massa pode ser muito rápida e alcançar pH 5,3 em menos de uma hora.

A velocidade na redução do $\mathrm{pH}$ da massa para valores inferiores a 5,3 tornou-se muito importante sob o ponto de vista de segurança alimentar, visto que deve ser alcançado em intervalo de tempo suficiente para inibir o crescimento de microrganismos como E. coli e S. aureus (BACUS, 1986; AMIF, 1997). Segundo a AMIF (1997), o processo pode ser julgado aceitável se o produto atingir pH 5,3 em tempo inferior a 28 horas quando a temperatura máxima de fermentação for menor ou igual a $35^{\circ} \mathrm{C}$.

$\mathrm{O}$ pH 5,3 foi alcançado imediatamente nos embutidos adicionados de GDL e vinho, enquanto que nos embutidos adicionados de cultura foi necessário tempo de fermentação de pouco mais de 10 horas. Desta forma, os tempos em que ambos os embutidos alcançaram pH 5,3 encontram-se bem aquém dos níveis máximos recomendados para garantir a segurança do produto.

Os valores de $\mathrm{pH}$ dos embutidos fermentados observados neste experimento aproximaram-se dos valores $(4,6$ a 5,2) citados por PEARSON e GILLET (1999) para salames cozidos. A massa com GDL apresentou pH de 4,81 no final da fermentação, enquanto que na massa adicionada de cultura o pH final foi de 4,37. Esses valores são semelhantes aos observados por KELLER e ACTON (1974) no final da fermentação de embutidos inoculados com cultura liofilizada e congelada após 48 e 24 horas 
(4,8 e 4,6 respectivamente). WARDLAW et al. (1973) também encontraram valores de pH próximos de 4,85 após 36 horas de fermentação em embutidos a base de carne de suínos e bovinos. Já ANDRADE et al. (2005) observaram, após 21 horas de fermentação, pH final próximo de 5,3 e 5,2 para embutidos adicionados de cultura em meio líquido e de cultura liofilizada, respectivamente. Em produtos fermentados cozidos a base de carne suína em que foram utilizados ácidos (cítrico e lático) encapsulados, TERRA, FRIES e TERRA (2004) encontraram nos produtos acabados valores de pH entre 5,10 e 5,28. NASSU et al. (2001) constataram valores de pH entre 5,05 e 5,14 em salames contendo diferentes proporções de carne caprina, suína e bovina após 15 dias de maturação.

A quantidade de ácido lático observada, após 26 horas de fermentação, foi de 1,03\% nos embutidos adicionados de GDL e de 1,26\% nos embutidos adicionados de cultura iniciadora. Esses valores enquadraram-se na faixa de 0,5 e 1,5\% de ácido lático, normalmente reportada para embutidos fermentados semi-secos (ACTON et al., 1972). REIS e SOARES (1998) constataram percentuais de ácido lático entre 0,96 e 1,15\% após 48 horas de fermentação e entre 1,25 e 1,31\% no final do período de maturação/secagem (270 horas). Também MATOS et al. (2005a) encontraram valores entre 1,40 e 1,50\% de ácido lático, após período de 48 horas de fermentação

Embora os embutidos elaborados por MATOS et al. (2005a) tenham apresentado altos valores de acidez em 48 horas de fermentação, sem adição de culturas ou acidulantes à massa, os valores de $\mathrm{pH}$ nunca alcançaram 5,3. Já os embutidos avaliados neste experimento apresentaram quantidades menores de ácido, mas a queda de pH chegou a valores próximos de 4,6 em 26 horas. Essa variação na quantidade de ácidos produzidos e valores de $\mathrm{pH}$ observados pode ser oriunda dos diferentes $\mathrm{pK}_{\mathrm{a}}$ dos ácidos orgânicos formados durante a fermentação, o que promove maior ou menor dissociação de prótons no meio.

Nos embutidos adicionados de cultura, a quantidade de ácidos formados após a fermentação foi da ordem de 0,6\%, expresso em ácido lático. Essa quantidade está de acordo com $0,47 \%$ observado por WARDLAW et al. (1973) e com 0,8 a 0,9\% encontrados por KELLER e ACTON (1974) e MATOS et al. (2005a) durante a etapa de fermentação. A acidificação da massa ocorreu muito rapidamente nos embutidos adicionados de GDL e vinho, provavelmente devido aos ácidos orgânicos pré-formados presentes no vinho e à formação do ácido glucônico a partir da hidrólise da GDL. Deve-se salientar que nesses embutidos houve queda na quantidade de ácidos nas primeiras 6 horas (Figura 1). É provável que nesse período tenha ocorrido metabolização de ácidos orgânicos por bactérias a outros ácidos e/ou a compostos neutros. Exemplo disso é a possível conversão do ácido glucônico a ácido acético por bactérias como L. plantarum e L. sake (TERRA, 1998).

A produção de ácidos orgânicos por outras bactérias, especialmente as láticas homofermentativas, podem ter contribuído para o aumento da acidez da massa nas últimas 20 horas (embora a acidez final no produto adicionado de GDL não tenha alcançado os valores iniciais).

A atividade de água foi reduzida a 0,92 no embutido adicionado de GDL e vinho e a 0,93 no embutido adicionado de cultura. Esses valores são próximos de 0,94, relatado por ANDRADE et al. (2005), mas acima dos observados por TERRA, FRIES e TERRA (2004) para embutidos cozidos adicionados de ácido cítrico e ácido lático encapsulados (0,89 e 0,90 respectivamente). NASSU et al. (2001) encontraram valores entre 0,90 e 0,92 para salames contendo diferentes proporções de carne caprina, suína e bovina, os quais foram afetados pelo tipo e proporção de carne utilizada.

A redução na atividade de água, oriunda da perda de água do embutido durante o seu processamento, deve-se à significativa redução na quantidade de proteínas miofibrilares solúveis causada pela redução do pH (WARDLAW et al., 1973) e à ação do calor na etapa de cozimento.

Segundo LEISTNER e RÖDEL (1975), produtos cárnicos com atividade de água menor que 0,95 e pH menor ou igual a 5,3 são considerados estáveis em temperatura ambiente. Para os valores de atividade de água e de pH obtidos neste experimento, ambos os embutidos (adicionado de GDL ou de cultura) não precisam ser estocados ou comercializados sob refrigeração.

A Tabela 2 apresenta os resultados da avaliação objetiva da cor e textura nos produtos acabados. 
TABELA 2 - PARÂMETROS FíSICOS DE QUALIDADE DE EMBUTIDOS FERMENTADOS COZIDOS A BASE DE CARNE OVINA

\begin{tabular}{lcc}
\hline & \multicolumn{2}{c}{ Tipo de fermentação } \\
\cline { 2 - 3 } Parâmetros & $\begin{array}{c}\text { Induzida } \\
\text { quimicamente } \\
\text { (GDL + vinho) }\end{array}$ & $\begin{array}{c}\text { Controlada } \\
\text { (Cultura iniciadora) }\end{array}$ \\
\hline Cor Objetiva & 46,85 & 46,76 \\
Luminosidade $\left(L^{*}\right)$ & 12,42 & 12,66 \\
Îndice de vermelho $\left(a^{\star}\right)$ & 8,62 & 7,75 \\
Índice de amarelo $\left(b^{*}\right)$ & 15,13 & 14,84 \\
Índice de saturação $\left(C^{*}\right)$ & 34,57 & 31,43 \\
Ângulo de tonalidade $\left(h^{\star}\right)$ & 3,15 & referência \\
Diferença global de cor $\left(\Delta E^{\star}\right)^{1}$ & & \\
Textura Objetiva & 1,16 & 1,50 \\
Resistência ao corte $(\mathrm{kgf} / \mathrm{cm})$ & $\mathrm{ND}$ & $\mathrm{ND}$ \\
Fraturabilidade $(\mathrm{kgf})$ & 17,81 & 0,38 \\
Dureza $(\mathrm{kgf})$ & 0,32 & 0,09 \\
Coesividade & 0,01 & 1,00 \\
Adesividade $(\mathrm{kgf} / \mathrm{mm})$ & 1,00 & 42,52 \\
Elasticidade & 34,16 & \\
Mastigabilidade $(\mathrm{kgf} / \mathrm{mm})$ & & \\
\hline
\end{tabular}

${ }^{1}$ diferença global da cor do embutido em relação à referência. ${ }^{2}$ segundo BOURNE (2002).

ND = não-detectado.

Os valores de luminosidade $\left(L^{*}\right)$ e índice de vermelho $\left(a^{\star}\right)$ ficaram ligeiramente acima dos reportados por CAVENAGHI et al. (2001) para salame cozido comercial $\left(L^{*}=40,59\right.$ e $\left.a^{*}=13,49\right)$ e por ELIAS et al. (2003) para embutido fermentado semi-seco $\left(L^{*}=39,20\right.$ e $\left.a^{*}=14,53\right)$ inoculado por diferentes tipos de culturas iniciadoras e maturado por 8 dias. Possivelmente, os maiores valores observados para $L^{*}$ neste experimento deve-se ao menor tempo de maturação de forma que a perda de água do produto é menor, favorecendo a sua luminosidade. Os pigmentos de mioglobina e nitrosomioglobina formados são concentrados de acordo com a perda água, conferindo cor vermelha rosada mais escura ao salame. Da mesma forma, os menores valores de $a^{*}$ observados foram, possivelmente, causados pelo menor tempo de maturação dos embutidos avaliados.

A redução dos valores de $L^{*}$ e o aumento dos valores de $a^{*}$ com a maturação são consistentes com os resultados reportados por ELIAS et al. (2003), que encontraram menores valores de $L^{*}$ $(38,75)$ e maiores valores de $a^{*}(16,43)$ para embutidos maturados por 30 dias. Também são condizentes com os dados reportados por CAVENAGHI et al. (2001) para salames "tipo Italiano", produto fermentado seco produzido geralmente com 30 dias de maturação. Esses autores encontraram valores médios de 37,70 para a luminosidade e de 14,55 para o índice de vermelho.

O baixo valor de $\mathrm{pH}$ dos embutidos também pode ter contribuído para os menores valores de $\mathrm{a}^{*}$ do que os reportados na literatura, pois valores abaixo de 4,9 têm sido considerados como prejudiciais para o desenvolvimento da cor em produtos curados (DEMEYER, 2002).

Embora o índice de amarelo $\left(b^{\star}\right)$ tenha se apresentado próximo do valor de 9,08 reportado por ELIAS et al. (2003) para embutidos semi-secos, os valores observados estão bem acima de 3,75 para salame cozido e pouco acima de 6,11 para salames secos, encontrados por CAVENAGHI et al. (2001) ao avaliarem os diversos tipos de salames comerciais. O valor de $b^{\star}$ de embutidos fermentados (aparentemente) aumenta com a etapa de maturação, haja vista os maiores valores de índice de amarelo $\left(b^{\star}=13,6\right)$ reportados por ELIAS et al. (2003) para embutidos secos. A diferença observada entre os valores de $b^{*}$, especialmente para os dados apresentados por CAVENAGHI et al. (2001), pode ser oriunda de variações na forma de processamento, como o uso de diferentes culturas, tempo, temperatura e umidade relativa de maturação, entre outras. 
Com relação às diferentes formas de fermentação, o uso da GDL ou da cultura iniciadora não influenciou os valores de $L^{*}$ ou $a^{*}$ para embutidos fermentados cozidos. Esse comportamento difere do observado para embutidos fermentados crus, cuja utilização de culturas iniciadoras favorece a formação e estabilização da cor do produto final (FREY, 1983). Atribuiu-se a melhora na coloração de embutidos fermentados secos pela ação de culturas iniciadoras ao favorecimento da redução de nitrito a nitrato, aumentando a disponibilidade de óxido nitroso (NO) para reagir com a mioglobina, e à ação catalase de alguns gêneros como Micrococcus e Staphylococcus. Bactérias lácticas heterofermentativas acumulam peróxido de hidrogênio no meio de crescimento, cujo acúmulo pode afetar adversamente as características sensoriais do produto cárnico. Provocam alterações oxidativas nas gorduras, rancidez e defeitos na coloração como o aparecimento indesejável de coloração verde (FREY, 1983; TERRA, 1998). No caso do embutido cozido é provável que pouca fermentação tenha ocorrido na massa adicionada de GDL, o que se traduziu em maior pH final. Desta forma, a catalase natural da carne foi suficiente para degradar o possível peróxido formado.

Ao contrário de $L^{*}$ e $a^{*}$, o índice de amarelo foi afetado pelo tipo de fermentação utilizada. Menores valores de $b^{*}$ para embutidos adicionados de culturas iniciadoras podem ser justificados pela observação de ELIAS et al. (2003) de que processos lipolíticos, mais intensos em produtos inoculados, causam mudanças na gordura presente nesses produtos. A gordura suína apresenta tonalidade amarelada, o que justifica a redução dos valores de $b^{*}$ nos embutidos adicionados de cultura iniciadora.

Maior índice de amarelo para embutidos adicionados de GDL causou elevação na saturação $\left(C^{*}\right)$ da cor desses produtos que tendeu para o laranja, traduzida no aumento do ângulo de tonalidade $\left(h^{\star}\right)$. Essas mudanças foram suficientes para gerar diferença global de cor $\left(\Delta E^{\star}\right)$ de 3,15, o que para PRÄNDL et al. (1994) indica percepção subjetiva da diferença de cor entre as amostras de "clara" a "muito clara" e, portanto, facilmente percebida pelo consumidor.

Os embutidos adicionados de cultura apresentaram maior dureza e resistência ao corte (Tabela 2), o que indica melhor consistência (firmeza) do que os embutidos adicionados de GDL. A fatiabilidade e firmeza do embutido cárnico ocorrem pela combinação da formação do gel, devido à coagulação das proteínas solubilizadas pelo sal. Essa coagulação, seja por acidificação ou aquecimento, envolve a formação de agregados mais estáveis e intensos, associados com a liberação de água. O gel formado é estabilizado pela liberação de água, que ocupa espaços entre os agregados e forma matriz que envolve gorduras e tecidos conectivos, determinando a textura dos embutidos (DEMEYER, 2002). Taxas elevadas de acidificação aumentam a taxa de secagem (FREY, 1983), determinando também o desenvolvimento da textura. Segundo DEMEYER (2002) o desenvolvimento da textura durante a fermentação é determinado pela queda do $\mathrm{pH}$, desconsiderando-se pequenas alterações no peso, enquanto que mudanças na textura durante a secagem é determinada apenas pela perda de água. É provável que a maior consistência observada nos embutidos adicionados de cultura seja decorrente da maior queda de $\mathrm{pH}$, o que também pode ter acarretado maior perda de água, favorecendo a textura.

Maior firmeza também favoreceu a mastigabilidade observada nas amostras adicionadas de cultura. A mastigabilidade pode ser definida como o número necessário de mordidas em força constante para reduzir a amostra até consistência aceitável para que seja engolida (BOURNE, 1978; SZCZESNIAK, 1998). Embora a adesividade tenha sido maior nesses produtos, os embutidos adicionados de GDL apresentaram-se ligeiramente mais coesos, o que contrasta com a diferença de firmeza entre as amostras.

Da mesma forma que observado por HARRIS e SHORTHOSE (1988) em amostras de carne, o "fator de elasticidade" (flexibilidade) em produtos fermentados cozidos parece desnecessária. Isto porque a razão entre a distância para se detectar o pico de deformação no segundo ciclo de compressão e a distância para o primeiro ciclo de compressão foi unitária. Também é coerente com a não-detecção do(s) pico(s) de fraturabilidade, uma vez que não se espera que o produto venha a se despedaçar (esmigalhar), quebrar ou estilhaçar durante a aplicação de força. 


\section{CONCLUSÃO}

Os dados obtidos indicaram a possibilidade de processar embutidos fermentados cozidos a base de carne ovina capazes de serem estocados e comercializados sem a necessidade de refrigeração e sem acarretar maiores riscos para a segurança alimentar. $\mathrm{O} \mathrm{pH}$ ideal para a conservação do produto foi alcançado mais rapidamente nos embutidos adicionados de GDL.

Os embutidos adicionados de cultura apresentaram-se mais ácidos, mais duros e com maior resistência ao corte, indicando melhor consistência (firmeza) do que os embutidos adicionados de GDL.

Os embutidos adicionados de GDL apresentaram tonalidade mais amarelada do que os adicionados de cultura, cuja diferença total de cor pode ser facilmente percebida subjetivamente.

\section{ABSTRACT \\ EFFECTS OF FERMENTATION TYPES IN FINAL QUALITY OF MUTTON COOKED FERMENTED SAUSAGES}

At this work was evaluated the effect of the controlled fermentation using starter culture and induced fermentation by adding glucono-delta-lactone (GDL) in the quality of cooked fermented mutton sausage. The GDL sausage initial $\mathrm{pH}$ value of 5.6 , decreased immediately to 5.3 after the addition of the ingredients, while the starter culture sausage ph needed 10 hours of fermentation to reach this value. After 26 hours of fermentation, the GDL sausage presentedl $\mathrm{pH}$ value of 4.81 and the acidity $1.03 \%$, as the starter culture sausage showed a final ph value of 4.37 and the acidity was $1.26 \%$. The products had a final water activity value between 0.92 (GDL) and 0.93 (culture). The yellow index was greater in GDL sausage $\left(b^{*}=8.62\right)$ than in culture sausage $\left(b^{*}=7.75\right)$. However, the lightness $\left(L^{*}=46.80\right)$ and the redness $\left(a^{*}=12.54\right)$ it wasn't affected by fermentation method. The culture sausages had greater hardness (20.38 vs $17.31 \mathrm{kgf}$ ) and shear resistance (1.50 vs $1.16 \mathrm{kgf} / \mathrm{cm}$ ) than GDL sausages, what indicates better consistency (firmness).

KEY-WORDS: FERMENTATION; SEMI-DRY SAUSAGE; SAUSAGE; MUTTON.

\section{REFERÊNCIAS}

1 ACTON, J.C.; WILLIANS, J.G.; JOHNSON, M.G. Effect of fermentation temperature on changes in meat properties and flavor of summer sausage. Journal of Milk Food Technology, v.35, p.264-268, 1972.

2 AMIF. American Meat Institute Foundation. Good manufacturing practices for fermented dry \& semi-dry sausage. Washington, DC, 1997.

3 AMSA. American Meat Science Association. Research guidelines for cookery, sensory evaluation, and instrumental tenderness measurements of fresh meat. Chicago: AMSA \& National Live Stock and Meat Board, 1995.

4 ANDRADE, J.C.; HAGUIWARA, M.M.H.; MIYAGUSKU, L.; YAMADA, E.A. Estudo da utilização de cultura starter na obtenção de produto cárneo embutido fermentado cozido. In: CONGRESSO DE CIÊNCIA E TECNOLOGIA DE ALIMENTOS, 3., 2005, São Pedro. Anais... São Pedro: CTC/ITAL, 2005. (CD-ROM)

5 AQUALAB. Analisador de atividade de água Decagon. Rio de Janeiro: ABRASEQ, 1997. 21 p. (Manual, 1).

6 BACUS, J.N. Utilization of microorganisms in meat processing. Letchworth: John Witley \& Sons, 1986. 170 p.

7 BERRY, B.W.; CROSS, H.R.; SMITH, G.C. Processing, chemical, sensory and physical properties of bacon-chopped and formed products, made from pork, beef, mutton and chevon. Journal of Muscle Foods, v.1, n.1, p.45-57, 1990.

8 BOURNE, M.C. Texture profile analysis. Food Technology, v.32, n.7, p.62-72, 1978.

9 Food texture and viscosity: concept and measurement. $2^{\text {nd }}$ ed. New York: Academic Press, 2002. 436 p.

10 BUSBOOM, J.R.; FIELD, R.A. Homemade, meat, poultry and game sausages. Washington: Washington State University, 2003. $14 \mathrm{p}$. 
12 CAVENAGHI, A.D.; BERAQUET, N.J.; CIPOLLI, K.M.V.A.B.; MELLO, R.L. Caracterização de salames tipo italiano tradicional e light e de embutido fermentado cozido fabricados no Brasil: Parte II - caracterização físicas e sensoriais. In: CONGRESSO BRASILEIRO DE CIÊNCIA E TECNOLOGIA DE CARNES, 2001, São Pedro. Anais... São Pedro: CTC/ ITAL, 2001. p.309-310.

13 DEMEYER, I.D. Quality control of fermented meat products. In: KERRY, J.; KERRY, J.; LEDWARD, D. (Eds.). Meat processing: improving quality. New York: CRC Press, 2002. p.359-393.

14 ELIAS, M.; MARINHO, A.; PALMA, V.; SANTOS, C.; ROSEIRO, C. The influence of the starter cultures in the production of regional Portuguese sausage - microbiological, physical and chemical properties. In: INTERNATIONAL CONGRESS OF MEAT SCIENCE AND TECHNOLOGY (ICOMST), 49., 2003, Campinas. Proceedings... Campinas, 2003. p.485-486.

15 EVERSON, C.E.; DANNER, W.E.; HAMMES, P.A. Improved starter culture for semidry sausage. Food Technology, v.24, p.42-44, 1970.

16 FREY, W. Fabricación fiable de embutidos. Zaragoza: Acribia, 1983. 194 p.

17 HARRIS, P.V.; SHORTHOSE, W.R. Meat texture. In: LAWRIE, R. (Ed.). Developments in meat science. London: Elsevier Applied Science, 1988. v.4. p. 245-296.

18 KELLER, J.E.; ACTON, J.C. Properties of a fermented semidry turkey sausage during production with lyophilized and frozen concentrates of Pediococcus cerevisiae. Journal of Food Science, v.39, p.836-840, 1974.

19 LEISTNER, L.; RÖDEL, W. The significance of water activity for microorganisms in meat. In: DUCKWORTH, R.B. (Ed.). Water reactions of foods. London: Academic Press, 1975. p.309-323.

20 MATOS, R.A.; MENEZES, C.M.; RAMOS, E.M.; RAMOS, A.L.S. Utilização de iogurte natural na fermentação de embutidos cozidos. In: SIMPÓSIO LATINO AMERICANO DE CIÊNCIA DOS ALIMENTOS (SLACA), 6., 2005, Campinas. Anais... Campinas: SBCTA, 2005a. (CD-ROM).

21 MATOS, R.A.; SAMPAIO, S.C.S.; RAMOS, E.M.; RAMOS, A.L.S. Avaliação de diferentes métodos de análise de acidez e pH em salames. In: COMPEX \& SEMINÁRIO DE INICIAÇÃO CIENTÍFICA, 9., 2005, Jequié. Anais... Jequié: UESB, 2005b. (CD-ROM).

22 MEDEIROS, J.X.; SANTO, E.E.; COUTO, F.A.; ARAUJO, F.C.; FREITAS, M.P.C.; CARDOSO, L.V.; AMARAL, E.S.; CARVALHO JÚNIOR, C.H.T. de; SCHTZ, R.G. Análise econômica da ovinocultura no Distrito Federal: sistemas de referência para apoio à tomada de decisão na cadeia produtiva produtores rurais e frigoríficos. Brasília: SEBRAE/DF, 2004. 89 p.

23 MOREIRA, J.N.; CORREIA, R.C.; ARAÚJO, J.R.; SILVA, R.R. Estudo do circuito de comercialização de carnes de caprinos e ovinos no eixo Petrolina-PE/Juazeiro-BA. Petrolina: EMBRAPA-CPATSA, 1997. 22 p.

24 NASSU, R.T.; GONÇALVES, L.A.G.; BESERRA, F.J.; FEITOSA, T. Estudo das características físico-químicas, microbiológicas e sensoriais de embutidos fermentados tipo salame formulados com diferentes proporções de carne caprina e suína. Boletim do CEPPA, v.19, n.2, p.243-256, 2001.

PEARSON, A.M.; GILlETT, T.A. Processed meats. $3^{\text {rd }}$ ed. New York: Chapman \& Hall, 1999. 664 p.

27 REIS, A.G.B.; SOARES, G.J.D. Salame colonial processado com carne suína e ovina. Revista Brasileira de Agrociência, v.2, n.2, p.115-120, 1998 . SAVIC, I.V. Small-scale sausage production. Rome: FAO, 1985. 94 p. (Food Animal and Health Paper, 52) SEBRAE/CE. Pesquisa sobre o consumo de carne ovina e caprina. Sobral: EMBRAPA-CAPRINOS, 2003.39 p. . Potencial de consumo de carnes de ovinos e caprinos em Fortaleza. Fortaleza, 1998. SEBRAE/RN. Diagnóstico da cadeia produtiva agroindustrial da caprinovinocultura do Rio Grande do Norte. Natal, 2001. p. 146. SZCZESNIAK, A.S. Sensory texture profiling - historical and scientific perspectives. Food Technology, v.52, n.8, p.54-57, 1998. TERRA, A.B.M.; FRIES, L.M.; TERRA, N.N. Particularidades na fabricação do salame. São Paulo: Editora Varela, 2004.152 p. TERRA, N.N. Apontamentos de tecnologia de carnes. São Leopoldo: Editora Unisinos, 1998. 216 p. WARDLAW, F.B.; SKELLEY, G.C.; JOHNSON, M.G.; ACTON, J.C. Changes in meat components during fermentation, heat processing and drying of a summer sausage. Journal of Food Science, v.38, p.1228-1231, 1973. TROPICAL BRASILEIRA, 1., 1994, Sobral. Anais... Sobral: EMBRAPA-SPI, 1994. p.115-128. 